\title{
Automated Antenna Design with Evolutionary Algorithms
}

\author{
Gregory S. Hornby \\ hornby@email.arc.nasa.gov \\ University of California Santa Cruz, Mailtop 269-3, NASA Ames Research Center, Moffett Field, CA
}

Al Globus

Derek S. Linden

San Jose State University

JEM Engineering, 8683 Cherry Lane, Laurel, Maryland 20707

Jason D. Lohn

NASA Ames Research Center, Mail Stop 269-1, Moffett Field, CA 94035

\begin{abstract}
Whereas the current practice of designing antennas by hand is severely limited because it is both time and labor intensive and requires a significant amount of domain knowledge, evolutionary algorithms can be used to search the design space and automatically find novel antenna designs that are more effective than would otherwise be developed. Here we present automated antenna design and optimization methods based on evolutionary algorithms. We have evolved efficient antennas for a variety of aerospace applications and here we describe one proof-of-concept study and one project that produced flight antennas that flew on NASA's Space Technology 5 (ST5) mission.
\end{abstract}

\section{Introduction}

The current practice of designing and optimizing antennas by hand is limited in its ability to develop new and better antenna designs because it requires significant domain expertise and is both time and labor intensive. As an alternative, researchers have been investigating evolutionary antenna design and optimization since the early 1990s, ${ }^{1-3}$ and the field has grown in recent years as computer speed has increased and electromagnetics simulators have improved. This techniques is based on evolutionary algorithms (EAs), a family stochastic search methods, inspired by natural biological evolution, that operate on a population of potential solutions using the principle of survival of the fittest to produce better and better approximations to a solution. Many antenna types have been investigated, including antenna arrays ${ }^{4}$ and quadrifilar helical antennas. ${ }^{5}$ In addition, evolutionary algorithms have been used to evolve antennas in-situ, ${ }^{6}$ that is, taking into account the effects of surrounding structures, which is very difficult for antenna designers to do by hand due to the complexities of electromagnetic interactions. Most recently, we have used evolutionary algorithms to evolve an antenna for the three spacecraft in NASA's Space Technology 5 (ST5) mission ${ }^{7}$ and are working on antennas for other upcoming NASA missions, such as one of the Tracking and Data Relay Satellites (TDRS). In the rest of this paper we will discuss our work on evolving antennas for both the ST5 and the TDRS missions. 


\section{Evolved X-band Antenna for NASA's ST5 Mission}

NASA's Space Technology 5 (ST5) mission is part of the New Millennium Program and its goal is to launch multiple miniature spacecraft to test, demonstrate and flight qualify innovative concepts and technologies in the harsh environment of space for application to future space missions. The ST5 mission consists of three miniaturized satellites, called micro-sats, which measure the effects of solar activity on the Earth's magnetosphere over a period of three months. The micro-sats are approximately $53 \mathrm{~cm}$ across, $48 \mathrm{~cm}$ high and, when fully fueled, weigh approximately 25 kilograms. Each satellite has two antennas, centered on the top and bottom of each spacecraft. Images of the ST5 spacecraft are shown in Fig. 1.

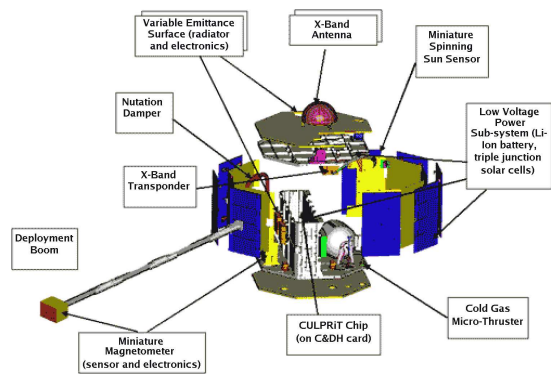

(a)

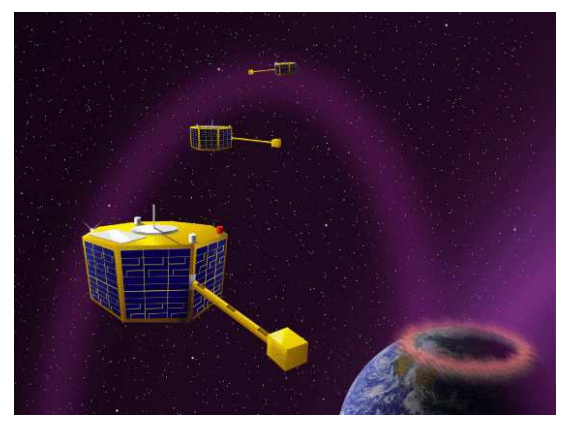

(b)

Figure 1. Artist's depiction of: (a) the spacecraft model showing the different spacecraft components, and (b) the ST5 mission with the three spacecraft in their string of pearls orbit.

The three ST5 spacecraft were originally intended to orbit in a "string of pearls" constellation configuration in a highly elliptical, geosynchronous transfer orbit that was set at approximately $35,000 \mathrm{~km}$ above Earth, with the initial requirements for their communications antennas as follows. The gain pattern must be greater than or equal to $0 \mathrm{dBic}$ (decibels as referenced to an isotropic radiator that is circularly polarized) at $40^{\circ} \leq \theta \leq 80^{\circ}$ and $0^{\circ} \leq \phi \leq 360^{\circ}$ for right-hand circular polarization. The antenna must have a voltage standing wave ratio (VSWR) of under 1.2 at the transmit frequency $(8470 \mathrm{MHz})$ and under 1.5 at the receive frequency $(7209.125 \mathrm{MHz})$. At both the transmit and receive frequencies the input impedance should be 50 $\Omega$. The antenna was restricted in shape to a mass of under $165 \mathrm{~g}$, and to fit in a cylinder of height and diameter of $15.24 \mathrm{~cm}$.

However, while our initial evolved-antenna was undergoing flight-qualification testing, the mission's orbital vehicle was changed, putting it into a much lower earth orbit and changing the specifications for the mission. The additional specification consisted of the requirement that the gain pattern must be greater than or equal to $-5 \mathrm{dBic}$ at $0^{\circ} \leq \theta \leq 40^{\circ}$.

To produce an initial antenna for the ST5 mission we selected a suitable class of antennas to evolve, configured our evolutionary design systems for this class, and then evolved a set of antenna designs that met the requirements. With minimal changes to our evolutionary system, mostly in the fitness function, we were able to evolve new antennas for the revised mission requirements and, within one month of this change, a new antenna was designed and prototyped.

\section{II.A. Initial Evolutionary Antenna Design Systems}

To meet the initial design requirements it was decided to constrain our evolutionary design to a monopole wire antenna with four identical arms, with each arm rotated $90^{\circ}$ from its neighbors. To produce this type of antenna, the EA evolves a description of a single arm and evaluates these individuals by building a complete antenna using four copies of the evolved arm. 
To encode a single arm of the antenna, the representation that we used consists of an open-ended, generative representation for "constructing" an arm. This generative representation for encoding antennas is an extension of our previous work in using a linear-representation for encoding rod-based robots. ${ }^{8}$ Each node in the tree-structured representation is an antenna-construction operator and an antenna is created by executing the operators at each node in the tree, starting with the root node. In constructing an antenna the current state (location and orientation) is maintained and operators add wires or change the current state. The operators are as follows: forward (length, radius), add a wire with the given length and radius extending from the current location and then change the current state location to the end of the new wire; rotate-x(angle), change the orientation by rotating it by the specified amount (in radians) about the $\mathrm{x}$-axis; rotate-y (angle), change the orientation by rotating it by the specified amount (in radians) about the y-axis; and rotate-z (angle), change the orientation by rotating it by the specified amount (in radians) about the z-axis.

An antenna design is created by starting with an initial feedwire and adding wires. The initial feed wire was set to start at the origin with a length of $0.4 \mathrm{~cm}$ along the Z-axis. In addition the radius of the wire segments was fixed at the start of a run, with all wire segments in all antenna designs having the same radius. To produce antennas that are four-way symmetric about the Z-axis, the construction process is restricted to producing antenna wires that are fully contained in the positive XY quadrant and then after construction is complete, this arm is copied three times and these copies are placed in each of the other quadrants through rotations of $90^{\circ} / 180^{\circ} / 270^{\circ}$.

The fitness function used to evaluate antennas is a function of the VSWR and gain values on the transmit and receive frequencies. The gain component of the fitness function uses the gain (in dBic) in $5^{\circ}$ increments about the angles of interest - from $40^{\circ} \leq \theta \leq 90^{\circ}$ and $0^{\circ} \leq \phi \leq 360^{\circ}$ - and consists of a gain error

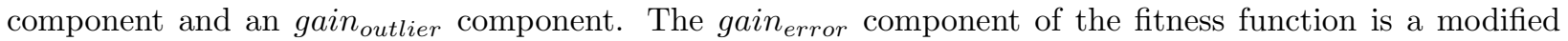
version of the Least Squares Error function, and was later modified to evolve the antenna for the revised

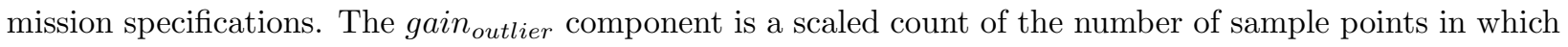
the gain value is below the minimum acceptable. The VSWR component of the fitness function is constructed to put strong pressure toward evolving antennas with receive and transmit VSWR values below the required amounts of 1.2 and 1.5, reduced pressure at a value below these requirements (1.15 and 1.25) and then no pressure to go below 1.1.

The three components are multiplied together to produce the overall fitness score of an antenna design:

$$
F=v s w r \times \text { gain }_{\text {error }} \times \text { gain }_{\text {outlier }}
$$

The objective of the EA is to produce antenna designs that minimize $F$.

\section{II.B. Revised Evolutionary Antenna Design Systems}

The new mission requirements required us to modify both the type of antenna we were evolving and the fitness functions we were using. The original antennas we evolved for the ST5 mission were constrained to monopole wire antennas with four identical arms but, because of symmetry, this four-arm design has a null at zenith and is unacceptable for the revised mission. To achieve an antenna that meets the new mission requirements the revised antenna design space we decided to search consists of a single arm. In addition, because of the difficulties we experienced in fabricating branching antennas to the required precision, we constrained our antenna designs to non-branching ones. Finally, because the satellite is spinning at about $40 \mathrm{RPM}$, it is important that the antennas have a uniform gain pattern in azimuth and so we dropped the gain $_{\text {outlier }}$ component of the fitness function and replaced it with a gain $_{\text {smoothness }}$ component. These three components are multiplied together to produce the overall fitness score of an antenna design, which is to be minimized:

$$
F=v s w r \times \text { gain }_{\text {error }} \times \text { gain }_{\text {smoothness }}
$$


For the revised fitness function the VSWR component was kept the same but changes were made to

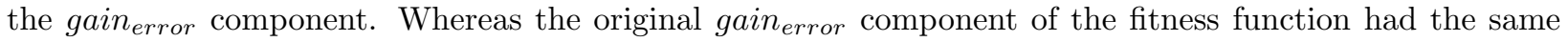
weighting and target gain value for each elevation angle, the revised gain component allows for a different target gain and weight for each elevation:

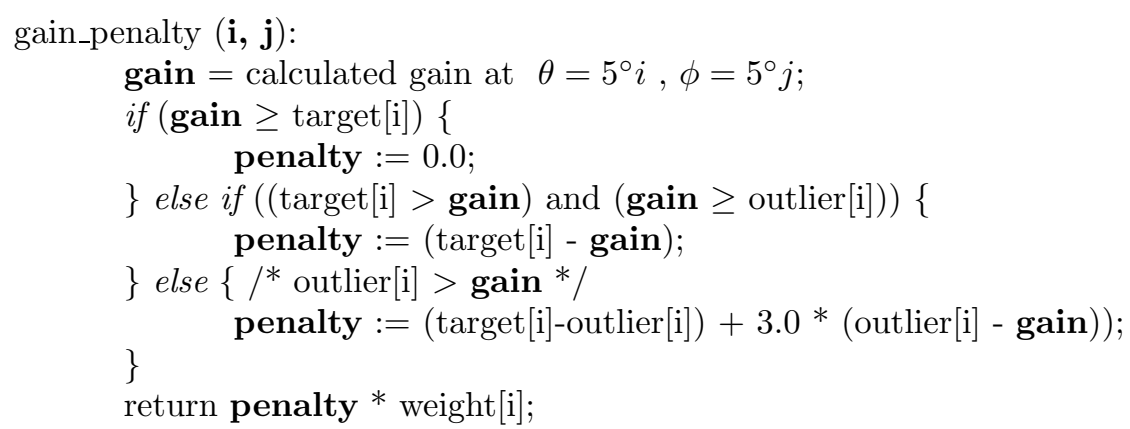

Target gain values at a given elevation are stored in the array target [] and are $2.0 \mathrm{dBic}$ for $i$ equal from 0 to 16 and $-3.0 \mathrm{dBic}$ for $i$ equal to 17 and 18. Outlier gain values for each elevation are stored in the array outlier [] and are $0.0 \mathrm{dBic}$ for $i$ equal from 0 to 16 and $-5.0 \mathrm{dBic}$ for $i$ equal to 17 and 18 . Each gain penalty is scaled by values scored in the array weight []. For the low band the values of weight [] are 0.1 for $i$ equal to 0 through 7; values 1.0 for $i$ equal to 8 through 16; and 0.05 for $i$ equal to 17 and 18 . For the high band the values of weight [] are 0.4 for $i$ equal to 0 through 7 ; values 3.0 for $i$ equal to 8 through 12 ; 3.5 for $i$ equal to $13 ; 4.0$ for $i$ equal to $14 ; 3.5$ for $i$ equal to $15 ; 3.0$ for $i$ equal to 16 ; and 0.2 for $i$ equal to 17 and 18. The final gain component of the fitness score is the sum of gain penalties for all angles.

To put evolutionary pressure on producing antennas with smooth gain-patterns around each elevation, the third component in scoring an antenna is based on the standard deviation of gain values. This score is a weighted sum of the standard deviation of the gain values for each elevation $\theta$. The weight value used for a given elevation is the same as is used in calculating the gain penalty.

\section{II.C. Results on ST5}

To meet the initial mission specifications we performed numerous runs of evolution, and selected from these the best antenna design, ST5-3-10, for fabrication and testing, Fig. 2.(a). This antenna met the initial mission requirements and was on track to be used on the mission until the mission's orbit was changed. After modifying our system to address the revised requirements we evolved antenna, ST5-33-142-7, Fig. 2.(b). In total, it took less than one month to modify our software and evolve this second antenna design, for which compliancy with mission requirements was confirmed by testing in an anechoic test chamber at NASA Goddard Space Flight Center. On March 22, 2006 the ST5 mission was successfully launched into space using the evolved antenna ST5-33-142-7 as one of its antennas. This evolved antenna is the first computer-evolved antenna to be deployed for any application and is the first computer-evolved hardware in space.

In comparison with traditional design techniques, the evolved antenna has a number of advantages in regard to power consumption, fabrication time, complexity, and performance. Originally the ST5 mission managers had hired a contractor to design and produce an antenna for this mission. Using conventional design practices the contractor produced a quadrifilar helix antenna (QHA). In Fig. 3 we show performance comparisons of our evolved antennas with the conventionally designed QHA on an ST5 mock-up. Since two antennas are used on each spacecraft - one on the top and one on the bottom - it is important to measure the overall gain pattern with two antennas mounted on the spacecraft. With two QHAs $38 \%$ efficiency was achieved, using a QHA with an evolved antenna resulted in $80 \%$ efficiency, and using two evolved antennas resulted in $93 \%$ efficiency. Lower power requirements result from achieving high gain across a wider range of elevation angles, thus allowing a broader range of angles over which maximum data 


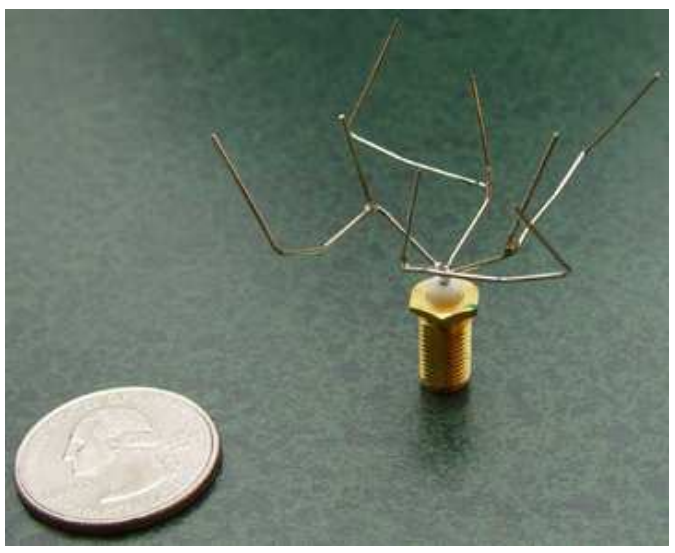

(a)

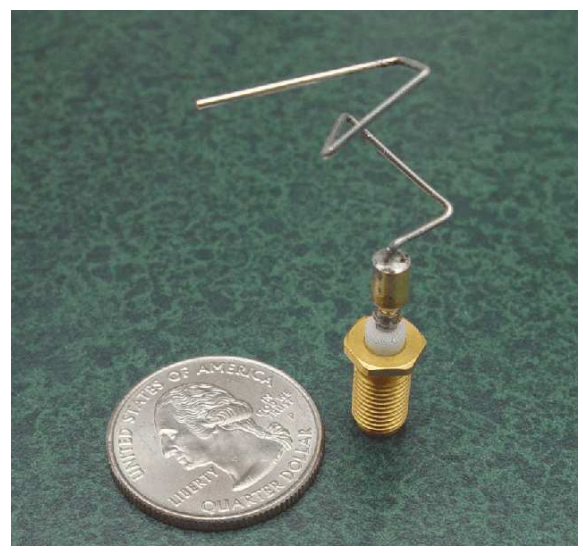

(b)

Figure 2. Photographs of prototype evolved antennas: (a) the best evolved antenna for the initial gain pattern requirement, ST5-3-10; (b) the best evolved antenna for the revised specifications, ST5-33-142-7.

throughput can be achieved. Since the evolved antenna does not require a phasing circuit, less design and fabrication work is required, and having fewer parts may result in greater reliability. In terms of overall work, the evolved antenna required approximately three person-months to design and fabricate whereas the conventional antenna required approximately five months. Lastly, the evolved antenna has more uniform coverage in that it has a uniform pattern with only small ripples in the elevations of greatest interest $\left(40^{\circ}-80^{\circ}\right)$. This allows for reliable performance as the elevation angle relative to the ground changes.

\section{S-band Antenna for TDRS-C}

In our most recent project we have evolved an S-band phased array antenna element design that meets the requirements of NASA's TDRS-C communications satellite. ${ }^{9}$ This mission is scheduled for launch early next decade and the original specifications called for two types of elements, one for receive only and one for transmit/receive. Using a combination of an evolutionary algorithm and a stochastic hill-climber we were able to evolve a single element design that meets both specifications thereby simplifying the antenna and reducing testing and integration costs.

TDRS-C is designed to carry a number of antennas, including a 46 element phased array. Element spacing is triangular at approximately $2 \lambda$. Each element gain must be $>15 \mathrm{dBic}$ on the boresight and $>10 \mathrm{dBic}$ to $\theta=20^{\circ}$ off boresight with both polarizations. For $\theta>30^{\circ}$, gain must be $<5 \mathrm{dBic}$. Axial ratio must be $\leq 5 \mathrm{~dB}$ over the field of view $\left(0-20^{\circ}\right)$. The receive-only element bandwidth covers $2200-2300 \mathrm{MHz}$ and the

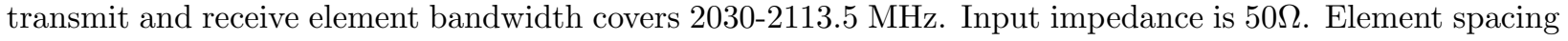
determines maximum footprint and there is no maximum height in the specification, although minimizing height and mass is a design goal. The combination of a fairly broad bandwidth, required efficiency and circular polarization at high gain makes for another challenging design problem.

\section{III.A. EA Configuration for TDRS-C}

We constrained our evolutionary design to a crossed-element yagi antenna. The element nearest the spacecraft is slightly separated and these two wires can be fed in such a way as to create circular polarization in either sense. All crossed-elements, including the first, are spaced and sized by evolution.

For this antenna problem, the representation we used to encode an antenna consists of a fixed length list of floating point numbers $\left(X_{i}\right)$. All $X_{i}$ are in the interval $0-1$ to simplify the variation operators. 


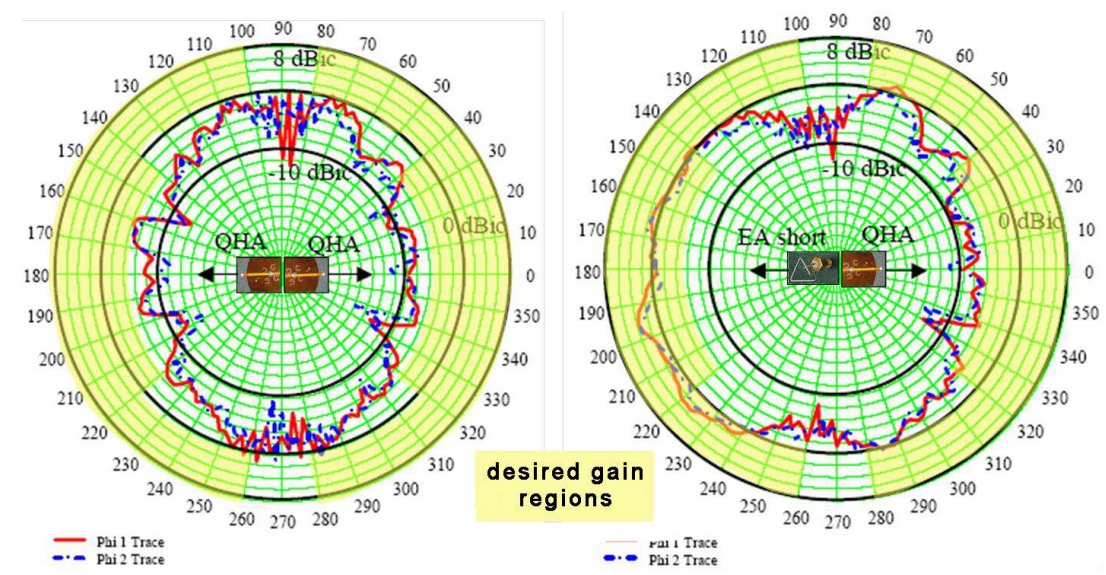

Figure 3. Measured patterns on ST-5 mock-up of two QHAs and a ST5-104.33 with a QHA. Phi $1=0$ deg., Phi $2=$ 90 deg.

Antenna parameters are determined from $X_{i}$ by linear interpolation within an interval chosen to generate reasonable parameters. $X_{1}$ determines the height of the antenna within the interval $3 \lambda-4 \lambda$ at the lowest frequency $(2030 \mathrm{MHz})$. The remaining pairs $\left(\left(X_{2 n+1}, X_{2 n+2}\right), n \geq 0\right)$ determine the size and spacing of each crossed-element (including the first, separated one). $X_{2 n+1}$ determines the spacing between elements and $X_{2 n+2}$ determines the size of the cross. For the first element, this is the absolute size of the cross in the interval $0.001 \lambda-1.5 \lambda$. For the remaining elements this is from the interval $0.8 s-1.2 s$ where $s$ is the size of the previous element.

Antennas fitness is a function of the standing wave ratio (VSWR) and gain values at 2030, 2075, 2120, 2210, 2255, and $2300 \mathrm{MHz}$. This fitness function to minimize is:

$$
\sum_{f} r m s\left(3, v_{f}\right)^{5}+r m s\left(1.5, v_{f}\right)+r m s\left(1.0, v_{f}\right)+\min \left(0,15.25-g_{f_{0}}\right)+\min \left(0,10.25-g_{f_{20}}\right)
$$

where $r m s(t, v)$ is the root mean square of a value above a target value $t, v_{f}$ is the VSWR at frequency $f$, $g_{f_{0}}$ is the gain at the boresight, and $g_{f_{20}}$ is gain $20^{\circ}$ off boresight. Note that a VSWR value above three is severely punished and improvements are always rewarded. Gain at the boresight and $20^{\circ}$ off bore sight is encouraged until it clears with a safety factor since simulation is never completely accurate. Side lobe minimization is not explicitly encouraged but this is achieved as a side effect of high gain near the boresight.

\section{III.B. TDRS-C Results}

Unlike our work in evolving an antenna for the ST5 mission, to evolve an antenna for TDRS-C we settled on using a three stage procedure for producing antenna designs. In the first stage, approximately 150 steady state evolutionary algorithm processes were run for up to 50,000 evaluations each with many parameters randomized (e.g., population size, number of crossed-elements, variation operators). In the second stage the best antenna from each of these runs was used as a start point for a stochastic hill climbing process with randomized mutation variation operators. These processes ran for up to 100,000 evaluations each. In the third and final stage the 23 best antennas from the second stage were subjected to another hill climbing procedure of up to 100,000 evaluations. All three of these stages were executed using the JavaGenes ${ }^{10}$ general purpose, open source stochastic search code written in Java and developed at NASA Ames. In addition, the Numerical Electromagnetics Code, Version 4 (NEC4) was used to evaluate all antenna designs. ${ }^{11}$

By the end of the third stage of computer-automated optimization most of the 23 designs subjected to this process were very close to meeting the specifications, and one antenna design exceeded them. The

\section{6 of 8}




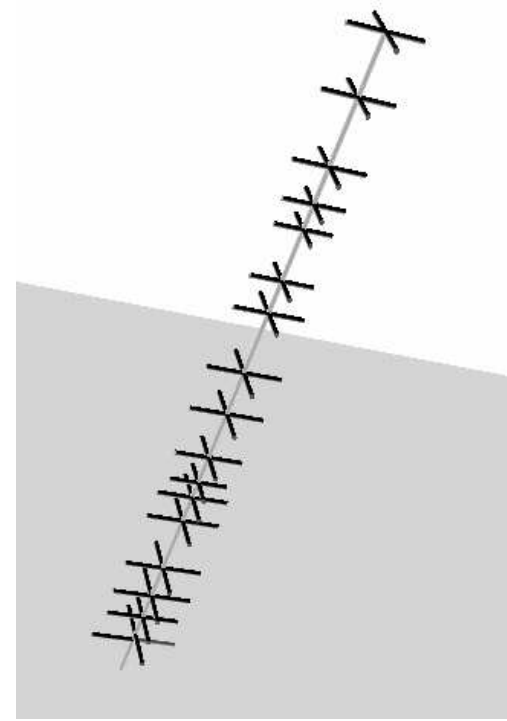

(a)

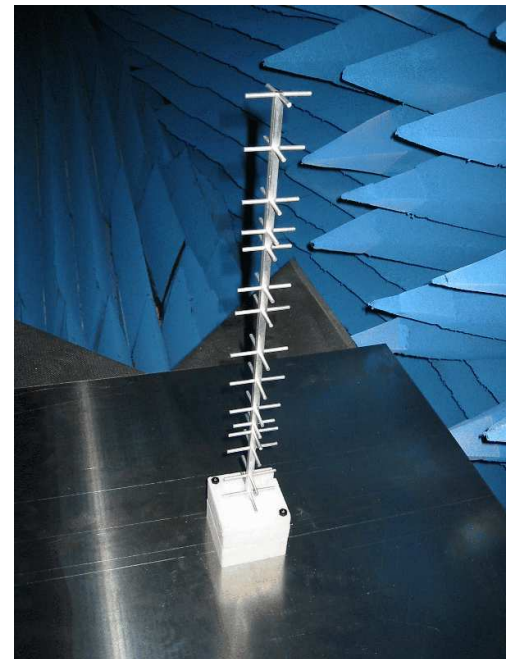

(b)

Figure 4. Best evolved TDRS-C antenna: (a) simulation and (b) fabricated.

one design that exceeded the mission specifications was subjected to further analysis by a more accurate electromagnectis software, WIPL-D version 5.2. Here, the design underwent some minor tuning through another evolutionary algorithm process and this final antenna design was then fabricated and tested. The results are largely consistent with the simulation. Gain and S1,1 plots are shown in Fig. 5. From here, it is up to mission managers whether they will select this antenna, or a human designed one, for use on this mission.

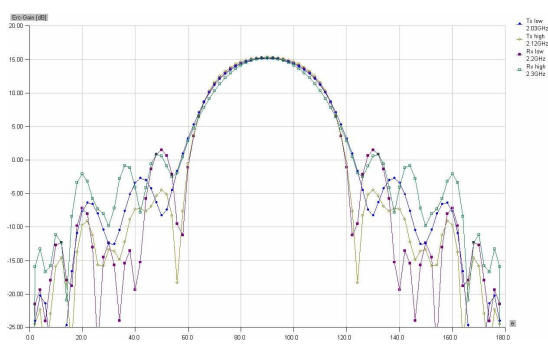

(a)

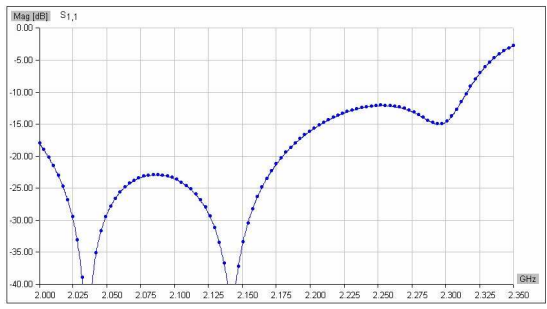

(b)

Figure 5. Results for the best evolved antenna for the TDRS-C mission: (a) Gain pattern with $90^{\circ}$ is on boresight; and (b) $\mathrm{S} 1,1$.

\section{Conclusion}

In this paper we have described our work in evolving antennas for two NASA missions. For both the ST5 mission and the TDRS-C missions it took approximately three months to set up our evolutionary algorithms and produce the initial evolve antenna designs. With the change in mission requirements for 
the ST5 mission it took roughly 4 weeks to evolve antenna ST5-33.142.7, and we expect that should such a change in requirements occur for the TDRS-C mission that we could produce a new antenna design that meets the revised specifications in under a month. Our approach has been validated with the successful launch on March 22, 2006 of the ST5 spacecraft and its successful operation throughout the lifetime of the mission.

In addition to being the first evolved hardware in space, our evolved antennas demonstrate several advantages over the conventionally designed antennas and manual design in general. The evolutionary algorithms we used were not limited to variations of previously developed antenna shapes but generated and tested thousands of completely new types of designs, many of which have unusual structures that expert antenna designers would not be likely to produce. By exploring such a wide range of designs EAs may be able to produce designs of previously unachievable performance. For example, the best antennas we evolved achieve high gain across a wider range of elevation angles, which allows a broader range of angles over which maximum data throughput can be achieved and may require less power from the solar array and batteries. With the evolutionary design approach it took approximately 3 person-months of work to generate the initial evolved antennas versus 5 person-months for the conventionally designed antenna and when the mission orbit changed, with the evolutionary approach we were able to modify our algorithms and re-evolve new antennas specifically designed for the new orbit and prototype hardware in 4 weeks. The faster design cycles of an evolutionary approach results in less development costs and allows for an iterative "what-if" design and test approach for different scenarios. This ability to rapidly respond to changing requirements is of great use to NASA since NASA mission requirements frequently change. As computer hardware becomes increasingly more powerful and as computer modeling packages become better at simulating different design domains we expect evolutionary design systems to become more useful in a wider range of design problems and gain wider acceptance and industrial usage.

\section{Acknowledgments}

This work was supported by NASA's CICT Program (contract AIST-0042) and by the Intelligent Systems Program. We thank the NAS facility for time on the Columbia 10,000 processor supercomputer.

\section{References}

${ }^{1}$ Haupt, R. L., "An Introduction to Genetic Algorithms for Electromagnetics," IEEE Antennas ES Propagation Mag., Vol. 37, April 1995, pp. 7-15.

${ }^{2}$ Michielssen, E., Sajer, J.-M., Ranjithan, S., and Mittra, R., "Design of Lightweight, Broad-band Microwave Absorbers Using Genetic Algorithms," IEEE Trans. Microwave Theory \&3 Techniques, Vol. 41, No. 6, June/July 1993, pp. $1024-1031$.

${ }^{3}$ Rahmat-Samii, Y. and Michielssen, E., editors, Electromagnetic Optimization by Genetic Algorithms, Wiley, 1999.

${ }^{4}$ Haupt, R. L., "Genetic Algorithm Design of Antenna Arrays," IEEE Aerospace Applications Conf., Vol. 1, Feb. 1996, pp. 103-109.

${ }^{5}$ Lohn, J. D., Kraus, W. F., and Linden, D. S., "Evolutionary Optimization of a Quadrifilar Helical Antenna," IEEE Antenna 83 Propagation Society Mtg., Vol. 3, June 2002, pp. 814-817.

${ }^{6}$ Linden, D. S., "Wire Antennas Optimized in the Presence of Satellite Structures using Genetic Algorithms," IEEE Aerospace Conf., April 2000.

7 "Space Technology 5 Mission," http://nmp.jpl.nasa.gov/st5/.

${ }^{8}$ Hornby, G. S., Lipson, H., and Pollack, J. B., "Generative Representations for the Automatic Design of Modular Physical Robots," IEEE Transactions on Robotics and Automation, Vol. 19, No. 4, 2003, pp. 703-719.

${ }^{9}$ Teles, J., Samii, M. V., and Doll, C. E., "Overview of TDRSS," Advances in Space Research, Vol. 16, 1995 , pp. 67-76.

${ }^{10}$ Globus, A., Crawford, J., Lohn, J., and Pryor, A., "Scheduling Earth Observing Satellites with Evolutionary Algorithms," Conference on Space Mission Challenges for Information Technology (SMC-IT), 2003.

${ }^{11}$ Burke, G. J. and Poggio, A. J., "Numerical Electromagnetics Code NEC-Method of Moments," Tech. Rep. UCID18834, Lawrence Livermore Lab, Jan 1981. 